\title{
Development of an Arabic Version of the Hiroshima University- Dental Behavioral Inventory: Cross-cultural Adaptation, Reliability, and Validity
}

\author{
Dany Daou ${ }^{1}$, Khaldoun Rifai ${ }^{2}$, Bassel Doughan ${ }^{1, *}$, Mounir Doumit $^{1}$ \\ ${ }^{1}$ Department of Public Health Dentistry, School of Dentistry, Lebanese University, Hadath, Beirut, Lebanon \\ ${ }^{2}$ Prosthodontics Department, School of Dentistry, Lebanese University, Hadath, Beirut, Lebanon
}

ARTICLE IN F O
Article History
Received 16 December 2017
Accepted 2 June 2018
Keywords
Arabic
oral health attitudes/behaviour
reliability
validity

\begin{abstract}
The Hiroshima University-Dental Behavioral Inventory (HU-DBI) has not been adequately translated to the Arabic language. The aim of this study is the translation and cultural adaption of the English HU-DBI into standard Arabic. The English HU-DBI was translated into Arabic by three bilingual dental academics. A nonmedical professional performed backward translation into English. The three bilinguals compared both English versions and accordingly modified the Arabic version. English and Arabic versions were answered by 58 bilingual participants. Focus groups and cognitive interviews were conducted to pretest the working version, after which the final Arabic version was tested for test-retest validity on 58 dental students. Bilingual validity and test-retest validity were assessed using the kappa statistic. Responses were similar in the English and Arabic versions for bilingual individuals and on both tests in the test-retest sample. Kappa values ranged from 0.85 to 1.00 for bilingual validity and between 0.82 and 1.00 for test-retest reliability. In conclusion, the Arabic HU-DBI will enable the assessment of knowledge and attitudes toward oral diseases and their prevention in Arab countries and will allow meaningful comparisons between different countries in the region.
\end{abstract}

(c) 2018 Atlantis Press International B.V. This is an open access article under the CC BY-NC license (http://creativecommons.org/licenses/by-nc/4.0/).

\section{INTRODUCTION}

The Hiroshima University-Dental Behavioral Inventory (HU-DBI), developed by Kawamura in 1988 [1], has been shown to be useful for the assessment of oral health attitudes and behaviour and has effectively been utilized for cross-cultural comparisons [2]. Among other languages, the questionnaire has been successfully translated to English, French, Italian, Korean, and Chinese [3]. Both the original Japanese version and the later developed English version have shown good test-retest reliability [1,4]. The English version, which also displayed good translation validity [5], has been largely used in different countries.

Oral health awareness in Arab countries is generally poor, and dental health education is lacking. Moreover, little is known regarding the population's knowledge and attitudes toward oral diseases and their prevention in comparison to other countries [6]. In a multinational comparison study using the HU-DBI, no Arabicspeaking countries were involved [3].

Although the utility of English as a second language is increasing in many Arabic speaking countries, socioeconomic and geographic disparities remain to create significant inequalities in English literacy. Therefore, although the English version of the HU-DBI may

*Corresponding author. Email: bassel.doughan@gmail.com theoretically be used, the use of a non-native language to respond to a questionnaire may be suboptimal because of language and cultural barriers. There is thus a need for the translation of the HU-DBI into the Arabic language to allow researchers to assess oral health knowledge in Arab countries. Although the various Arab countries each use different dialects, standard Arabic is the official dialect utilized in writing and in official communication and is understood by all Arab populations.

The HU-DBI has been utilized in several Arab countries (Saudi Arabia, Jordan, Sudan, UAE), but the majority of studies have used the English version [7-10]. Although one recent study has used an Arabic translation from English [8], the translation does not appear to have been subjected to adequate validity testing according to recommended guidelines $[11,12]$. The aim of the present study was the translation, cultural adaptation, and validation of the HU-DBI into standard Arabic.

\section{MATERIALS AND METHODS}

\subsection{Questionnaire}

This cross-sectional validation study consisted of the following parts: (1) forward translation from English to Arabic; (2) backward translation from Arabic to English; (3) adaptation of an Arabic working version; (4) assessment of bilingual reliability 
(Arabic-English); (5) focus groups and cognitive interviewing; and (6) assessment of test-retest reliability (Arabic-Arabic). The study was approved by the local scientific committee, and the patients and students who participated in this study gave their informed consent. All questionnaires were completed anonymously, and participants were given the option of discontinuing at any time.

\subsection{Translation}

The process was performed according to the traditional standard recommendations for cross-cultural adaptation through four separate steps (World Health Organization recommendations) [12]. The English version of the HU-DBI questionnaire (Appendix 1) was submitted to a committee of three bilingual dental academics from the Faculty of Dentistry (two from the department of Dental Oral Health and one prosthodontist) who were all familiar with the terminology covered by the questionnaire and trained in the use of the instrument. The emphasis was to select a committee of bilingual members whose mother tongue is Arabic, who are fluent in both spoken and written English and who are experienced in teaching using the English language.

The translators were asked to avoid literal translation, emphasizing the need for conceptual translation in order to reach the broadest target population. Each member initially gave a separate translated version, and then the three jointly gave one final translated Arabic version (forward translation). This Arabic version was then translated back into the English language by a nonmedical professional English translator (backward translation) who was blinded to the aim of the study. The translator was asked to perform the backward translation emphasizing on cultural and conceptual equivalence rather than on the linguistic aspect.

In the third step, the literal assessment and adaptation of the semantic equivalence was performed by the original three bilingual translators in keeping with the aim of the study. The two English versions of the questionnaire (the original and the backward translated one) were compared, and discrepancies were discussed and resolved by agreement through modifying words and expressions in the Arabic translation, as needed. The resulting final Arabic version was endorsed as the working questionnaire.

\subsection{Validation}

\subsubsection{Bilingual validity}

Bilingual validity was assessed on a sample of 58 bilingual participants recruited from patients coming for routine practice or minor interventions (decay-clinical examination-routine check-up, etc.). Inclusion criteria were: (1) age $>18$ years; (2) adequate cultural and educational levels to understand health questionnaires (completion of at least high school education); and (3) fluency in both the Arabic and English languages. Patients were asked to rank their degree of proficiency in each of the two languages on a scale of $0-10$, and only patients with a rating of 7 or above in both languages were eligible for inclusion. All participants were informed that participation was on a voluntary basis. After signing an informed consent form, they were asked to answer the English version and then the Arabic version of the HU-DBI separately at different times (with an interval of approximately 24 hours).

\subsubsection{Pretesting and cognitive interviewing}

The working questionnaire was tested for face validity using both focus groups and cognitive interviewing in order to detect any potential conceptual problem. An experienced academic researcher in the field performed the task. Respondents were 15 postgraduate dental students from the Lebanese University who were randomly chosen on a voluntary basis from different age groups, different socioeconomic backgrounds, and from both genders. The respondents were asked to answer the questions and each was discussed separately to confirm whether each question or word was correctly understood. Accordingly, the panel expert committee concluded that no modifications were necessary and approved the Arabic translated version.

\subsubsection{Test-retest validity}

Sample size calculation based on the responses in bilingual testing indicated that at least 50 participants would be required to detect kappa values of 0.9 and confidence intervals of \pm 0.1 . A group of 58 dental students were selected on a voluntary basis. After signing an informed consent form, they were asked to answer the Arabic version of the HU-DBI. They were recalled 24 hours later to answer the same Arabic inventory.

\subsection{Statistical Analysis}

Descriptive statistics for the proportion of participants responding positively to each question were generated. Intra-rater agreement for bilingual validity and for test-retest validity was assessed by calculating the kappa statistic, 95\% confidence interval, and $p$-value for each question separately. Data were processed using the Statistical Package for Social Sciences (SPSS, version 20.0, IBM and Stata/SE 11.1. Statistical significance was set at 0.05 .

\section{RESULTS}

\subsection{Bilingual Reliability}

Percentages of positive response were generally similar in both English and Arabic questionnaires for the bilingual respondents (Table 1). More than $50 \%$ of the participants answered affirmatively to questions $1,3,9,13,14,15$, and 20 , whereas for all remaining questions the majority $(>50 \%)$ answered negatively. The largest proportion of participants agreed that they worry about having bad breath (question 13, 80.4\% and $82.6 \%$ in English and Arabic forms, respectively). By contrast, the lowest proportion of participants agreed that they worry about having gums that bleed when brushing (question 2, 13.0\% and $10.9 \%$ in English and Arabic forms, respectively).

Intra-rater agreement between the responses to the English and Arabic questionnaires was very good to excellent for all 20 questions (kappa values of 0.850-1.000; Table 1). The lowest kappa values were noted for the questions regarding the use of a childsized tooth brush, thinking that one cannot help having false teeth when old, and checking one's teeth in the mirror after brushing 
(questions 5, 6, and 12; kappa values $=0.850,0.858$, and 0.867, respectively). Questions 4, 9, 10,11, and 17, by contrast, had perfect agreement between the English and Arabic versions (kappa = 1.000 ), and overall, 13 out of the 20 questions displayed excellent kappa values of greater than 0.900 .

\subsection{Test-retest Reliability}

The student respondents provided very similar answers to the Arabic questionnaire on both tests (Table 2). More than $50 \%$ of the students answered affirmatively to questions $2,3,4,13,14,15,18$,

Table 1 Response rate and intra-rater reliability between English and translated Arabic version of the HU-DBI questionnaire $(n=46)$

\begin{tabular}{|c|c|c|c|c|c|}
\hline \multirow[t]{2}{*}{ Questions } & \multicolumn{2}{|c|}{ Response (Yes) $n(\%)$} & \multicolumn{3}{|c|}{$\begin{array}{l}\text { Bilingual intra-rater reliability } \\
\text { (English vs. Arabic) }\end{array}$} \\
\hline & English & Arabic & Kappa & $95 \% \mathrm{CI}$ & $p$-Value \\
\hline 1 I don't worry much about visiting the dentist & $34(74)$ & $32(70)$ & 0.893 & $(0.749-1.000)$ & $<0.001^{*}$ \\
\hline 2 My gums tend to bleed when I brush my teeth & $6(13)$ & $5(11)$ & 0.897 & $(0.698-1.000)$ & $<0.001^{*}$ \\
\hline 3 I worry about the colour of my teeth & $31(67)$ & $33(72)$ & 0.898 & $(0.759-1.000)$ & $<0.001^{*}$ \\
\hline 4 I have noticed some white deposits on my teeth & $16(35)$ & $16(35)$ & 1.000 & $(1.000-1.000)$ & $<0.001^{*}$ \\
\hline 5 I use a child sized toothbrush & $13(28)$ & $16(35)$ & 0.850 & $(0.687-1.000)$ & $<0.001^{*}$ \\
\hline 6 I think that I cannot help having false teeth when I am old & $17(37)$ & $16(35)$ & 0.858 & $(0.704-1.000)$ & $<0.001^{*}$ \\
\hline 7 I am bothered by the colour of my gums & $8(17)$ & $7(15)$ & 0.920 & $(0.767-1.000)$ & $<0.001^{*}$ \\
\hline 8 I think my teeth are getting worse despite my daily brushing & $12(26)$ & $11(24)$ & 0.942 & $(0.830-1.000)$ & $<0.001^{*}$ \\
\hline 9 I brush each of my teeth carefully & $31(67)$ & $31(67)$ & 1.000 & $(1.000-1.000)$ & $<0.001^{*}$ \\
\hline 10 I have never been professionally taught how to brush & $13(28)$ & $13(28)$ & 1.000 & $(1.000-1.000)$ & $<0.001^{*}$ \\
\hline 11 I think I can clean my teeth well without using toothpaste & $11(24)$ & $11(24)$ & 1.000 & $(1.000-1.000)$ & $<0.001^{*}$ \\
\hline 12 I often check my teeth in a mirror after brushing & $20(44)$ & $19(41)$ & 0.867 & $(0.721-1.000)$ & $<0.001^{*}$ \\
\hline 13 I worry about having bad breath & $37(80)$ & $38(83)$ & 0.928 & $(0.788-1.000)$ & $<0.001^{*}$ \\
\hline 14 It is impossible to prevent gum disease with tooth brushing alone & $29(63)$ & $27(59)$ & 0.909 & $(0.786-1.000)$ & $<0.001^{*}$ \\
\hline 15 I put off going to the dentist until I have a toothache & $27(59)$ & $29(63)$ & 0.909 & $(0.786-1.000)$ & $<0.001^{*}$ \\
\hline 16 I have used a dye to see how clean my teeth are & $5(11)$ & $6(13)$ & 0.897 & $(0.698-1.000)$ & $<0.001^{*}$ \\
\hline 17 I use a toothbrush which has hard bristles & $11(24)$ & $11(24)$ & 1.000 & $(1.000-1.000)$ & $<0.001^{*}$ \\
\hline 18 I don't feel I've brushed well unless I brush with strong strokes & $19(41)$ & $19(41)$ & 0.910 & $(0.789-1.000)$ & $<0.001^{*}$ \\
\hline 19 I feel I sometimes take too much time to brush my teeth & $17(37)$ & $16(35)$ & 0.953 & $(0.861-1.000)$ & $<0.001^{*}$ \\
\hline 20 I have had my dentist tell me that I brush very well & $20(44)$ & $21(46)$ & 0.956 & $(0.871-1.000)$ & $<0.001^{*}$ \\
\hline
\end{tabular}

"Statistically significant at $p<0.01$; Response (yes) refers to the number and percentage of participants responding "yes" to each question; CI, confidence intervals; HU-DBI, Hiroshima University-Dental Behavioral Inventory.

Table 2 Response rate and intra-rater reliability for test-retest of Arabic version of the HU-DBI questionnaire $(n=46)$

\begin{tabular}{|c|c|c|c|c|c|}
\hline Questions & \multicolumn{2}{|c|}{ Response (Yes) $n(\%)$} & \multicolumn{3}{|c|}{$\begin{array}{l}\text { Bilingual intra-rater reliability } \\
\text { (English vs. Arabic) }\end{array}$} \\
\hline & Test 1 & Test 2 & Kappa & $95 \% \mathrm{CI}$ & $p$-Value \\
\hline 1 I don't worry much about visiting the dentist & $10(17)$ & $12(21)$ & 0.888 & $0.737-1.000$ & $<0.001^{*}$ \\
\hline 2 My gums tend to bleed when I brush my teeth & $33(57)$ & $32(55)$ & 0.965 & $0.897-1.000$ & $<0.001^{*}$ \\
\hline 3 I worry about the colour of my teeth & $49(84)$ & $49(84)$ & 1.000 & $1.000-1.000$ & $<0.001^{*}$ \\
\hline 4 I have noticed some white deposits on my teeth & $35(60)$ & $34(59)$ & 0.893 & $0.720-0.992$ & $<0.001^{*}$ \\
\hline 5 I use a child sized toothbrush & $15(26)$ & $16(28)$ & 0.956 & $0.871-1.000$ & $<0.001^{*}$ \\
\hline 6 I think that I cannot help having false teeth when I am old & $20(34)$ & $20(34)$ & 1.000 & $1.000-1.000$ & $<0.001^{*}$ \\
\hline 7 I am bothered by the colour of my gums & $18(31)$ & $18(31)$ & 1.000 & $1.000-1.000$ & $<0.001^{*}$ \\
\hline 8 I think my teeth are getting worse despite my daily brushing & $18(31)$ & $19(33)$ & 0.960 & $0.883-1.000$ & $<0.001^{*}$ \\
\hline $9 \quad$ I brush each of my teeth carefully & $21(36)$ & $21(36)$ & 1.000 & $1.000-1.000$ & $<0.001^{*}$ \\
\hline 10 I have never been professionally taught how to brush & $25(43)$ & $25(43)$ & 1.000 & $1.000-1.000$ & $<0.001^{*}$ \\
\hline 11 I think I can clean my teeth well without using toothpaste & $13(22)$ & $14(24)$ & 0.952 & $0.858-1.000$ & $<0.001^{*}$ \\
\hline 12 I often check my teeth in a mirror after brushing & $27(47)$ & $27(47)$ & 1.000 & $1.000-1.000$ & $<0.001^{*}$ \\
\hline 13 I worry about having bad breath & $49(84)$ & $49(84)$ & 1.000 & $1.000-1.000$ & $<0.001^{*}$ \\
\hline 14 It is impossible to prevent gum disease with tooth brushing alone & $32(55)$ & $32(55)$ & 0.930 & $0.835-1.000$ & $<0.001^{*}$ \\
\hline 15 I put off going to the dentist until I have a toothache & $37(64)$ & $38(66)$ & 0.962 & $0.889-1.000$ & $<0.001^{*}$ \\
\hline 16 I have used a dye to see how clean my teeth are & $11(19)$ & $10(17)$ & 0.826 & $0.635-1.000$ & $<0.001^{*}$ \\
\hline 17 I use a toothbrush which has hard bristles & $27(47)$ & $27(47)$ & 0.965 & $0.898-1.000$ & $<0.001^{*}$ \\
\hline 18 I don't feel I've brushed well unless I brush with strong strokes & $37(64)$ & $36(62)$ & 0.889 & $0.767-1.000$ & $<0.001^{*}$ \\
\hline 19 I feel I sometimes take too much time to brush my teeth & $15(26)$ & $15(26)$ & 0.820 & $0.651-0.990$ & $<0.001^{*}$ \\
\hline 20 I have had my dentist tell me that I brush very well & $35(60)$ & $33(57)$ & 0.858 & $0.724-0.992$ & $<0.001^{*}$ \\
\hline
\end{tabular}

"Statistically significant at $p<0.01$; Response (yes) refers to the number and percentage of participants responding "yes" to each question; CI, confidence intervals; HU-DBI, Hiroshima University-Dental Behavioral Inventory. 
and 20 , whereas for all remaining questions the majority ( $>50 \%)$ answered negatively. Similar to the bilingual group, the largest proportion of students agreed that they worry about having bad breath (84\%). Interestingly, the lowest positive answers were for questions 1 and 16 ; only $17-21 \%$ of the students report that they do not to worry about visiting the dentist and only $17-19 \%$ had ever used a dye to disclose plaque on their teeth.

Intra-rater agreement between the repeated responses to the Arabic questionnaire was very good to excellent for all 20 questions (kappa values of 0.820-1.000; Table 2). The lowest kappa values were noted for the questions regarding the use of a disclosing dye and the feeling of taking too much time when brushing (questions 16 and 19; kappa $=0.826$ and 0.820, respectively). Questions 3, 6, 7, 9, 10, 12, and 13 , by contrast, had perfect test-retest agreement $(\mathrm{kappa}=$ 1.000 ), and overall, 14 out of the 20 questions displayed excellent kappa values of greater than 0.900 .

\section{DISCUSSION}

The aim of this study was the translation and cultural adaptation of the (HU-DBI) into standard Arabic. In line with recommended guidelines $[11,12]$, the process included translation, retranslation, semantic and literal adaptation, and pilot study validation on a target population. Focus group and cognitive interviewing ensured that all translated questions were being understood as intended by the authors of the original questionnaire. Testing in bilinguals confirmed understanding of the questions in responses in both English and Arabic forms. Finally, the high test-retest reliability scores achieved when using the final translated Arabic version attest to the excellent reliability of the translated instrument.

The vast majority of previous research using the HU-DBI questionnaire in Arab countries (Saudi Arabia, Jordan, Sudan, UAE, etc.) used the questionnaire in its English form [7-10]. Only one recent study used an Arabic version translated from English [8]. Although the questionnaire underwent forward and backward translation in addition to reliability testing in bilinguals, only two linguists were asked to answer the English and Arabic versions for reliability testing. It is very probable that the linguists' knowledge of English and Arabic languages is not representative of the general population's. Additionally, the researchers reported high percentage agreement and thus concluded a high level of reliability between the English and Arabic languages. Percentage agreement, however, is an unreliable method of testing reliability, and the kappa statistic is recommended instead [13].

In addition to our reporting of the recommended kappa values and confidence intervals, several other factors attest to the strength of the translation and adaptation process used in this study. Compared with previous reports of testing bilingual reliability on samples of 16-37 volunteers $[5,14-20]$, our data are based on 58 bilingual patients fluent in both Arabic and English. In an article discussing the sample size required for pilot studies, the recommended sample size for test-retest pilot studies is 35-40 [21].

Several methods have been described in the recruitment of bilingual samples, ranging from the use of Likert scales for self-rating [22] to asking the participants one short question about their ability to use each of the two languages [18]. The authors of the original HU-DBI report attached a question to the actual questionnaire and requested the participants to describe their proficiency in each of the two languages being assessed using one of the following answers: (1) no difficulty using the language in everyday life; (2) no difficulty using the language in business and professional writing/ reading; or (3) same as mother tongue. To ensure the selection of a truly bilingual sample, we opted for the first method and included only participants who rated their proficiency in both the English and Arabic languages with a score of at least 7 out of 10. In a first attempt without considering the degree of proficiency in English, results showed a large discrepancy in answers between the English and the Arabic versions. In this attempt, 10 questions out of 20 had an unsatisfactory kappa result $(<0.8)$. A focus group discussion was performed with eight participants (two dentists, two dental students, three patients, and one translator) and minor changes were introduced to the formulation of the above 10 mentioned questions. The second attempt was then successfully performed on a new group mastering the two languages leading to kappa results greater than 0.8 for all questions.

In line with what is commonly performed in similar studies, bilingual participants were asked to first answer the English version and then the translated Arabic version [3]. The time interval between answering the two questionnaires, however, is debatable. The longer the time interval, the less the recall bias but also the greater the chance that the second questionnaire is not answered (attrition of the sample and losing participants). In our study, and according to patients' convenience, time interval was at least 1 hour and up to 10 hours (same day), as recommended by several authors [17,20]. Although using a longer time interval may have reduced recall bias, it carries the risk that some habits may change if the person is affected by the information, especially in such a questionnaire regarding dental health.

Finally, focus groups and cognitive interviewing were carried out after ensuring that the reliability in bilinguals was high and that the translation is adequately accurate compared to the original language. The rationale behind this is that monolinguals do not necessarily behave as bilinguals. Bilinguals often experience cultural adaptation and may perceive an Arabic word in a way that is culturally similar to someone whose first language is English. Although in-depth personal interviews are one way of achieving this goal, we chose to organize focus groups in an attempt to improve the validity of our results because such methodology is based on open discussions which do not exist in individual interviews.

\section{CONCLUSION}

The HU-DBI was translated and adapted into the Arabic language. It was successfully tested for validity and reliability and showed high interrater reliability in both bilingual testing and in repeated Arabic testing. The translated Arabic inventory is believed to be a useful tool, necessary to fill the gap in the dental and oral health fields in Arab countries. The use of a validated Arabic version will enable the assessment of the knowledge and attitudes toward oral diseases and their prevention in Arab countries and will allow meaningful comparisons between these countries.

\section{CONFLICTS OF INTEREST}

None declared. 


\section{REFERENCES}

[1] Kawamura M. Dental behavioral science. The relationship between perceptions of oral health and oral status in adults. Hiroshima Daigaku Shigaku Zasshi 1988;20;273-86.

[2] Dagli RJ, Tadakamadla S, Dhanni C, Duraiswamy P, Kulkarni S. Self reported dental health attitude and behavior of dental students in India. J Oral Sci 2008;50;267-72.

[3] Komabayashi T, Kawamura M, Kim KJ, Wright FA, Declerck D, Goiâs Mdo C, et al. The hierarchical cluster analysis of oral health attitudes and behaviour using the Hiroshima University-Dental Behavioural Inventory (HU-DBI) among final year dental students in 17 countries. Int Dent J 2006;56;310-16.

[4] Kawabata K, Kawamura M, Miyagi M, Aoyama H, Iwamoto Y. The dental health behavior of university students and test-retest reliability of the HU-DBI. J Dent Health 1990;40;474-75 (in Japanese).

[5] Kawamura M, Kawabata K, Sasahara H, Fukuda S, Iwamoto Y. Dental behavioral science: Part IX. Bilinguals' responses to the dental behavioral inventory (HU-DBI) written in English and in Japanese. J Hiroshima Univ Dent Soc 1992;22;198-204.

[6] Kawas SA, Fakhruddin KS, Rehman BU. A comparative study of oral health attitudes and behavior between dental and medical students; the impact of dental education in United Arab Emirates. J Int Dent Med Res 2010;3;6-10.

[7] Rahman B, Kawas SA. The relationship between dental health behavior, oral hygiene and gingival status of dental students in the United Arab Emirates. Eur J Dent 2013;7;22-27.

[8] Khalid K, Naidoo S, Elamin E. Oral health behaviours and attitudes using the modified Arabic version of Hiroshima UniversityDental Behavioural Inventory (HU-DBI) among Sudanese dental students. Int J Dentistry Oral Sci 2016;3;326-30.

[9] Al-Wahadni AM, AL-Omiri MK, Kawamura M. Differences in self-reported oral health behavior between dental students and dental technology/dental hygiene students in Jordan. J Oral Sci 2004;46;191-97.

[10] Hashim R, Ibrahim M. Oral health attitudes and behavior among dental students in Ajman, United Arab Emirates. J Int Dent Med Res 2013;6;84-87.

[11] Sousa VD, Rojjanasrirat W. Translation, adaptation and validation of instruments or scales for use in cross-cultural health care research: a clear and user-friendly guideline. J Eval Clin Pract $2011 ; 17 ; 268-74$.

[12] World Health Organization. Process of translation and adaptation of instruments. Available from: http://www.who.int/substance_abuse/research_tools/translation/en/. 2009.

[13] Hallgren KA. Computing inter-rater reliability for observational data: an overview and tutorial. Tutor Quant Methods Psychol 2012;8;23-34.

[14] Badovinac A, Božić D, Vučinac I, Vešligaj J, Vražić D, Plančak D. Oral health attitudes and behavior of dental students at the University of Zagreb, Croatia. J Dent Educ 2013;77;1171-78.

[15] Peker K, Uysal O, Bermek G. Dental training and changes in oral health attitudes and behaviors in Istanbul dental students. J Dent Educ 2010;74;1017-23.

[16] Yildiz S, Dogan B. Self reported dental health attitudes and behaviour of dental students in Turkey. Eur J Dent 2011;5; 253-59.

[17] Jaramillo JA, Jaramillo F, Kador I, Masuoka D, Tong L, Ahn C, et al. A comparative study of oral health attitudes and behavior using the Hiroshima University-Dental Behavioral Inventory (HU-DBI) between dental and civil engineering students in Colombia. J Oral Sci 2013;55;23-28.

[18] Kawamura M, Yip HK, Hu DY, Komabayashi T. A cross-cultural comparison of dental health attitudes and behaviour among freshman dental students in Japan, Hong Kong and West China. Int Dent J 2001;51;159-63.

[19] Kawamura M, Spadafora A, Kim KJ, Komabayashi T. Comparison of United States and Korean dental hygiene students using the Hiroshima University-Dental Behavioural Inventory (HU-DBI). Int Dent J 2002;52;156-62.

[20] Polychronopoulou A, Kawamura M, Athanasouli T. Oral selfcare behavior among dental school students in Greece. J Oral Sci 2002;44;73-78.

[21] Hertzog MA. Considerations in determining sample size for pilot studies. Res Nurs Health 2008;31;180-91.

[22] Epstein J, Osborne RH, Elsworth GR, Beaton DE, Guillemin F. Cross-cultural adaptation of the Health Education Impact Questionnaire: experimental study showed expert committee, not back-translation, added value. J Clin Epidemiol $2015 ; 68 ; 360-69$. 
APPENDIX 1

HU-DBI questionnaire

\begin{tabular}{|c|c|c|}
\hline Questions & Agree & Disagree \\
\hline 1 I don't worry much about visiting the dentist. & $\square$ & $\square$ \\
\hline 2 My gum tends to bleed when I brush my teeth. & $\square$ & $\square$ \\
\hline 3 I worry about the color of my teeth. & $\square$ & $\square$ \\
\hline 4 I have noticed some white sticky deposits on my teeth. & $\square$ & $\square$ \\
\hline 5 I use a child sized toothbrush. & $\square$ & $\square$ \\
\hline 6 I think that I can't help having false teeth when I am old. & $\square$ & $\square$ \\
\hline 7 I'm bothered by the color of my gum. & $\square$ & $\square$ \\
\hline 8 I think my teeth are getting worse despite my daily brushing. & $\square$ & $\square$ \\
\hline 9 I brush each of my teeth carefully. & $\square$ & $\square$ \\
\hline 10 I have never been taught professionally how to brush. & $\square$ & $\square$ \\
\hline 11 I think I can clean my teeth well without using toothpaste. & $\square$ & $\square$ \\
\hline 12 I often check my teeth in a mirror after brushing. & $\square$ & $\square$ \\
\hline 13 I worry about having bad breath. & $\square$ & $\square$ \\
\hline 14 It is impossible to prevent gum disease with tooth brushing alone. & $\square$ & $\square$ \\
\hline 15 I put off going to dentist until I have a toothache. & $\square$ & $\square$ \\
\hline 16 I have used a dye to see how clean my teeth are. & $\square$ & $\square$ \\
\hline 17 I use a toothbrush which has hard bristles. & $\square$ & $\square$ \\
\hline 18 I don't feel I have brushed well unless I brush with hard strokes. & $\square$ & $\square$ \\
\hline 19 I feel I sometimes take too much time to brush my teeth. & $\square$ & $\square$ \\
\hline 20 I have had my dentist tell me that I brush very well. & $\square$ & $\square$ \\
\hline
\end{tabular}

